\title{
Thin Dielectric Film Thickness Determination by Advanced Transmission Electron Microscopy
}

A. C. Diebold ${ }^{1}$, B. Foran ${ }^{1}$, C. Kisielowski², D. Muller ${ }^{3}$, S. Pennycook ${ }^{4}$, E. Principe ${ }^{5}$, and S. Stemmer ${ }^{6}$

${ }^{1}$ International SEMATECH, Austin, TX

${ }^{2}$ National Center for Electron Microscopy, Berkeley, CA

${ }^{3}$ Bell Laboratories Lucent Technologies, Murray Hill, NJ

${ }^{4}$ Oak Ridge National Laboratories, Oak Ridge, TN

${ }^{5}$ Applied Materials, Santa Clara, CA

${ }^{6}$ Materials Department, University of California Santa Barbara, CA 
Abstract: High Resolution Transmission Electron Microscopy (HR-TEM) has been used as the ultimate method of thickness measurement for thin films. The appearance of phase contrast interference patterns in HR-TEM images has long been confused as the appearance of a crystal lattice by non-specialists. Relatively easy to interpret crystal lattice images are now directly observed with the introduction of annular dark field detectors for scanning TEM (STEM). With the recent development of reliable lattice image processing software that creates crystal structure images from phase contrast data, HR-TEM can also provide crystal lattice images. The resolution of both methods was steadily improved reaching now into the sub Angstrom region. Improvements in electron lens and image analysis software are increasing the spatial resolution of both methods. Optimum resolution for STEM requires that the probe beam be highly localized. In STEM, beam localization is enhanced by selection of the correct aperture. When STEM measurement is done using a highly localized probe beam, HR-TEM and STEM measurement of the thickness of silicon oxynitride films agree within experimental error. In this paper, the optimum conditions for HR-TEM and STEM measurement are discussed along with a method for repeatable film thickness determination. The impact of sample thickness is also discussed. The key result in this paper is the proposal of a reproducible method for film thickness determination.

Keywords: Thin films, HR-TEM, STEM, thickness determination. 


\section{Comparison of HR-TEM and ADF-STEM}

The thickness of silicon dioxide and silicon oxynitride films used as transistor gate dielectrics has fallen below $2 \mathrm{~nm}$. Due to the increase in leakage current and diffusion of boron from the highly doped polysilicon gate electrode through the gate dielectric into the channel, higher dielectric constant materials are being investigated as replacements for silicon dioxide and silicon oxynitride. Thicker films of a higher dielectric constant material match the capacitance of thin silicon dioxide layers. Development of new processes for deposition of these materials requires accurate, reproducible measurement of film thickness. In addition, accurate, reproducible measurement of the thickness of interfacial layers, such as (unintentional) $\mathrm{SiO}_{2}$ or silicate, is also critical. For these reasons, HR-TEM and ADF-STEM have been used to measure film thickness and interfacial properties. Initial comparisons of thickness measurement between these methods pointed to potential differences (Principe et al., 2001). In this paper, we show that both methods can provide thickness values that agree within experimental error when measurement conditions are optimized. The physics of imaging thin amorphous dielectric films between crystalline silicon is also described for both methods. A key result of this paper is the proposal of a reproducible method of measuring the thickness of thin dielectric films.

Although three different TEM imaging modes have been used in the characterization of thin oxide films (Diebold et al., 1999; Muller,1999 \& 2000; Muller\& Neaton 2001) only two of these provide silicon crystal lattice information that can be used as an internal calibration, HR-TEM and ADF-STEM. ADF STEM is also known as Z-contrast microscopy. TEM based electron holography can also provide crystal lattice information 
that can be used to calibrate the dimensions in an image reconstructed from a hologram (Rau \& Lichte, 1999). In HR-TEM, the electron beam simultaneously illuminates the entire imaged area. For atomic resolution the sample is oriented along a low index zone axis. The beam interacts strongly with the crystal, forming multiple diffracted beams. HR-TEM "lattice" images are formed when two or more of the diffracted beams interfere at the image plane to form an image. Lattice images do NOT depict the projected atom columns, instead, they are interference patterns of the directly transmitted beam with diffracted beams. The true column positions of the projected crystal lattice may correspond to intensity maxima or minima, or any other value depending on objective lens focus and sample thickness. The interference pattern can be used to calibrate the dimensions observed in the lattice image, and HR-TEM images obtained as "on-axis lattice fringe images" from the silicon substrate can be used as a high-resolution calibration of transistor feature sizes. Image formation in Scanning TEM (STEM) is different from the high resolution, phase contrast images discussed above.

ADF-STEM images are direct images of the crystal lattice. STEM images are formed by monitoring the transmitted electron intensity as a finely focused electron beam is scanned across a very thin electron transparent sample as in TEM. Images can be formed from both the change in intensity of the directly transmitted beam (bright field images) and from electrons scattered into large angles (dark field images). The latest generation STEM systems are equipped with high angle annular dark field (HA-ADF) detectors that provide very high image contrast at atomic resolution (Muller 2000; Muller \& Neaton, 2001; Howie, 1979). The HA-ADF STEM image is an amplitude image and not a phase contrast image. There is a one to one correspondence between the atom-like spots in HA- 
ADF-STEM image and the position of columns of atoms (Muller 2000; Pennycook 1997 \& Boatner, 1988; Pennycook \& Jesson 1990). In addition the form of the image does not vary strongly with defocus, or thickness, or lens conditions. Although a detailed and quantitative understanding of the contrast in these images requires consideration of subtle physics (Muller 2000; Perovic et al., 1993; Silcox et al., 1992), the essential reason for the intuitive nature of ADF imaging is that the annular detector averages over a wide range of scattering angles. The diffraction pattern falling on the annular detector may show complex interference effects but the total integrated signal is simply dependent on the total scattered intensity. The HA-ADF STEM image is therefore a map of the atomic scattering factor that is a strong function of the atomic number, hence the term Z-contrast imaging (Pennycook, 1997).

Fundamentally, the differences between the HRTEM lattice imaging and HA-ADF STEM arise because the first is a coherent image while the latter gives an incoherent image. In both images there is a definite relationship between object and image, but in a coherent image it depends sensitively on many factors such as specimen thickness and lens focus which makes it difficult to determine details of the specimen through simple intuitive interpretation. An incoherent image, such as that obtained through a regular photo camera, has a much simpler dependence on specimen and lens parameters. Extensive simulations or computation is not required to deduce useful information on interface position and roughness with useful accuracy and repeatability. An excellent example of the stability of the incoherent HA-ADF STEM image is given Muller (2000) who observed that film thickness, measured from cross section images, is not a function 
of sample thickness even up to $600 \mathrm{~nm}$ as shown in Figure 1. This information provides key motivation for the method we propose below.

Recently, Taylor et al., (2000) studied theoretically the HR-TEM accuracy for thickness measurements of $1.056 \mathrm{~nm}$ and $1.629 \mathrm{~nm}$ thin silicon dioxide films sandwiched between crystalline silicon. Using multi slice simulation, the effect of different sample thickness, defocus, tilt, and spherical aberrations of the objective lens on such measurements was investigated. This work concludes that thickness errors of $10 \%$ are common and that only a HRTEM with no spherical lens aberration could measure film thickness exactly if single lattice images are considered. This study provides considerable motivation for reducing lens aberration.

The technique of electron exit wave reconstruction removes much of the variability in the form of the bright field TEM image. By now, the focal series reconstruction is a reliable process that starts with a series of images of the same location taken under different focus conditions (Kisielowski et al., 2001; Coene et al., 1996; Thust et al., 1996; Jia \& Thust, 1999), and then combines them into a single phase and amplitude image of the electron exit wave. HR-TEM combined with focal-series reconstruction can produce direct images of the crystal structures with sub-Ångstrom resolution down to about 0.08 $\mathrm{nm}$ since the phase of the electron exit wave marks the position of the projected atomic columns and the resolution is improved. In fact, phase images of the electron exit wave and ADF STEM images depict the 1s ground state Bloch wave trapped on atom columns (van Dyck \& Chen, 1999).

Comparison of the spatial resolution of HR-TEM and HA-ADF-STEM depends on whether or not hardware and software corrections are used. Uncorrected HA-ADF-STEM 
can resolve features $50 \%$ smaller than uncorrected HR-TEM if the Scherzer resolution limit is considered. Nellist and Pennycook (1998) have pointed out that TEM and STEM have important differences in their sensitivity to energy spread in the electron beam near the limit of resolution. The important beams in STEM are traveling in equal and opposite angles through the lens and undergo the same phase change due to instability (energy spread). In HR-TEM, the beams forming the high resolution image travel at angles and then interfere with the direct beam to form the image. The beams traveling at angles have a different phase change from the direct beam. STEM images do not have the exponential damping envelope that HR-TEM images do when approaching the limits of resolution (Nellist \& Pennycook, 1998). Thus, it was necessary to reduce the energy spread in HR-TEM in order to obtain sub-Ångstrom information (O'Keefe et al., 2001; Kisielowski et al., 2001).

New lens technology is currently designed to actively correct for spherical aberration of the probe forming lens (Krivankek et al., 1999) or the objective lens (Haider et al., 1998). Traditionally, in the STEM field, the probe-forming lens has been called the objective lens, but in a TEM/STEM instrument the probe-forming lens is usually referred to as the condenser lens. This creates some confusion; in any case, in a STEM an aberration corrector is located before the specimen, whereas in a TEM it is after the specimen. To date, probe correction in STEM has resulted in resolutions of about 0.08 $\mathrm{nm}$ (Batson et al., 2002), which is close to the theoretically predicted limit of $\sim 0.05 \mathrm{~nm}$ (Pennycook et al., 2002). Objective lens correction with HR-TEM has been used to improve resolution to about $0.14 \mathrm{~nm}$ (Haider et al., 1998). Hardware correction of the three-fold lens aberrations in combination with exit wave reconstruction has also reached 
a resolution of about $0.08 \mathrm{~nm}$ (O'Keefe et al., 2001; Wang et al., 1999; Kisielowski et al., 2000). Although one might expect that microscopes of comparable performance may give identical dielectric thickness information on the same sample, this requires careful attention to details such as selection of aperture size for STEM measurement. Before discussing this point further, the impact of interfacial roughness and sample thickness (not film thickness) is further presented below.

\section{Interface Imaging and Sample Thickness}

It is important to note that physics of HR-TEM image formation is significantly different than that of HA-ADF-STEM discussed below. In HR-TEM, projection through the TEM sample thickness allows for lattice fringes to be observed even for micro-rough interfaces that can make the interface appear to be smooth as shown in Figure 2. In principle, it might seem that a single lattice image could be used to deduce the proper structure of the interface if thickness and defocus were chosen suitably and the image related to the crystal structure by theory. However, there is usually a lack of uniqueness involved in the process because of the a-priori unknown interface structure. In order to reproducibly measure the thickness of dielectric films between crystalline silicon or other crystalline materials, the effect of sample thickness must be reduced. Baumann, et al. (2000), have simulated the effect of interfacial roughness on single HR-TEM images to illustrate the complications in interpretation. The impact of the TEM sample thickness variation includes an apparent shift in HR-TEM determined oxide thickness of up to 0.3 $\mathrm{nm}$ for a surface roughness having magnitude of $1.1 \mathrm{~nm}$ peak to peak and a single period of $7.6 \mathrm{~nm}$ (Baumann et al., 2000). The simulation results of Bauman et al. are shown in Figure 3. In contrast, Muller has simulated electron exit waves and ADF-STEM imaging 
of thin oxide films and found that both methods should give the same result for film thickness when sample thickness is thin enough and the crystalline side of the interface remains ordered as one would expect. We show the simulated exit wave and an ADF STEM image for a $7.6 \mathrm{~nm}$ thick sample in Figure 4, and the line profiles through both images in Figure 5. The line profile through the exit wave image matches the line profile through the simulated ADF-STEM image when the sample thickness is $7.6 \mathrm{~nm}$. At larger thickness, HAADF -STEM and HR-TEM exhibit significant differences. We note here that with the introduction of electron exit wave reconstruction, the effort involved in the comparison of lattice images with simulation becomes comparable to that of ADF STEM in that extensive defocus and thickness series are not generally needed.

Interfacial imaging has been an issue for some time. Ross \& Stobbs (1991), studied the oxidation of single crystal silicon and the thickness of the interface before the widespread availability of HA-ADF STEM and focal series reconstruction. The Fresnel method was used to study interfacial properties. Fresnel fringes are seen at the interface of every crystal. A series of images are obtained at different defocuses. These studies concluded that a $0.5 \mathrm{~nm}$ layer with intermediate stoichiometry is seen at the silicon - silicon dioxide interface for both wet and dry oxidation methods. Ross also discusses the impact of sample tilt on the observed interfacial thickness. For example, for a $10 \mathrm{~nm}$ sample thickness, a tilt of 1 degree will result in a $0.17 \mathrm{~nm}$ observed extension of the crystalline region into the amorphous region (Ross \& Stobbs, 1991).

A fundamental part of thickness determination by ADF-STEM and HR-TEM is determining how many atoms and how much crystalline order is required for each method to detect an ordered column of atoms. Recently, Kisielowski, et al., (2001; 2002) 
compared the sensitivity of the HA-ADF-STEM with the HR-TEM. Under ideal conditions (absence of an amorphous layer) the sensitivity of the One Angstrom HRTEM allows for the detection of single $\mathrm{Si}(\mathrm{Z}=14)$ atoms, while the sensitivity of a TwoAngstrom HA-ADF-STEM was demonstrated to be at least good enough to detect single gold $(Z=79)$ atoms in thin samples with certainty. In the presence of correlated noise from an amorphous $\mathrm{SiO}_{2}$, however, the HR-TEM sensitivity is reduced and an extrapolation of these data indicates that both methods may have comparable detection limits in this case. One could expect that roughly $5 \mathrm{Si}$ atoms aligned in a column are required to generate a detectable signal above the noise levels but the atoms need not be next to each other in the sample. Muller has simulated electron exit waves and HA-ADFSTEM images of a thin sample $\sim 6 \mathrm{~nm}$ thick having interfacial roughness of the type where the atoms on the crystalline side of the interface were perfectly ordered. Since both methods depict the 1s ground state of the Bloch wave the interface is observed at the same location. Previous simulations without the exit wave reconstruction had shown that HR-TEM could underestimate silicon dioxide thickness by 0.3 to $0.6 \mathrm{~nm}$. Kisielowski's and Muller's most recent work shows that in the absence of disorder at the interface, exit wave reconstruction and ADF-STEM should observe the same film thickness for a well prepared thin sample of $\mathrm{Si} / \mathrm{SiO} 2 /$ poly $\mathrm{Si}$. However, both agree that HA-ADF-STEM will be more sensitive to chemical roughness such as diffusion of oxygen atoms into a column of Si atoms and disorder in the atomic columns. Muller points out that STEM images are very sensitive to sample tilt when imaging atomic columns. Thus disorder in a column reduces its contrast. The use of ultra-thin samples has reduced the potential impact of interfacial roughness and disorder in this study. 
Previously, Principe, et al., (2001; 2001b) used both HA-ADF STEM, HR-TEM with focal series image reconstruction, and Cs corrected HR-TEM to study the thickness of silicon oxynitride. In addition, XPS was used to determine both thickness and nitrogen concentration. TEM data was obtained using extremely thin samples in order to minimize issues associated with interfacial roughness. A comparison of HR-TEM images from different sample thickness' is shown in Figure 2. A comparison of HR-TEM, ADFSTEM, and XPS is shown in Figure 6. Preparation of $6 \mathrm{~nm}$ thick cross-sections is difficult and time consuming. Image reconstruction and HR-TEM equipped with aberration correction gave the same dielectric thickness. In this paper, we find that HRTEM and HA-ADF-STEM also measure the same thickness when the correct STEM aperture is selected. Aperture selection is discussed below in the section on Image Delocalization.

A further useful capability of the HA-ADF STEM is the possibility of assessing the state of the last few monolayers of Si below the oxide. Often, a bright band of contrast is seen which could be due to strain induced by oxygen or impurity diffusion, or might indicate true Z-contrast. In this case, the origin can be determined simply by increasing the inner angle of the ADF detector. Strain contrast will reverse whereas true Z-contrast will not. This is a simple way to test for diffusion of small amounts of heavy impurities such as Hf for example (Pantelides et al., 2001). In reconstructed exit waves strain can directly be determined by measuring local displacements of the columns to a precision approaching $1 \mathrm{pm}$.

Muller has shown that ADF STEM, EELS and synchrotron X-ray reflectivity all measure the same gate oxide thickness whereas uncorrected HR-TEM consistently 
underestimates the thickness by $1.9 \AA$. Part of this error arose from the image delocalization in HR-TEM where the last layer of silicon is not always the last layer of dots in the image (see fig 2a of Taylor et al., (2000). The amount of delocalization will vary depending on microscope and imaging conditions used, but exit wave reconstruction can can quantitatively correct for this problem.

\section{Plasmon Loss Imaging and layer Thickness}

The use of images formed from contrast changes in the plasmon tail made using energy filtering TEM has been recently proposed as a method for determining the thickness of thin dielectric films (Pantel et al., 2001). Muller has shown several issues with this method (Muller, 1998).

The first issue is that the elastic scattering contrast is preserved (and often dominates) inelastic images, unless diffraction contrast can be suppressed. In figure $3 \mathrm{~b}$ of (Wang, 1997), Wang shows a lattice image taken in the Al plasmon, with substantial intensity in an adjacent $\mathrm{Ti}$ crystal where there is no $\mathrm{Al}$ at all. Unless, a large series of tilted-beam images is averaged together, the energy filtered images are dominated by diffraction contrast and are thus seriously unreliable. Also, this technique is extremely dose inefficient (by a factor of 10) in a method that already was very dose inefficient to begin with. STEM avoids this problem by using a large collection angle.

The second issue is that plasmons are delocalized, and have tails that may extend $\sim 1 \mathrm{~nm}$, which can make it hard to locate the interface. The third issue is that bulk plasmons are suppressed at interfaces, interface modes appear at different energies, so a bulk mode cannot be used to locate an interface. This is shown in figure 5.24 of (Egerton, 1989). 


\section{Image Delocalization in STEM}

In addition to obtaining the phase of the exit wave, the through focal series reconstruction of HR-TEM images also provides the additional benefit that it corrects the image delocalization from the point-spread function (PSF) of the microscope. In principle, algorithms intended to improve noise and correct the PSF could be also applied to STEM images as well. This is rarely done as random scan distortions are often too large for signal averaging - it would blur out the atom positions. Image delocalization is significantly different in ADF-STEM, HR-TEM (lattice images), and reconstructed electron exit waves. Figure 7 illustrates delocalization in real space for ADF-STEM and lattice images (HR-TEM). In a properly optimized STEM, there is little to be gained by performing a linear deconvolution of a single image as the signal to noise ratio at high spatial frequencies is low and there are no contrast reversals to correct.

However, very few STEM instruments today are set up under conditions intended to minimize image delocalization. In many cases this is driven by the acceptance tests for the instrument that are based on producing a lattice image containing high spatial frequencies (e.g. $0.136 \mathrm{~nm}$ ) and a given (presumably large) beam current. The larger the probe-forming aperture (which provides a high-frequency cutoff in reciprocal space), the higher the spatial frequencies allowed in the image (although there is no guarantee that the higher frequencies can be focused to the same cross-over due to off-axis lens aberrations), and the higher the beam current. As the beam current increases as the square of the aperture size, even a $20 \%$ increase in aperture size, gives a $44 \%$ increase in beam current. The price paid for these increases is a dramatic decrease in image contrast (see Figure 8) at all spatial frequencies, and especially the silicon $\{111\}$ lattice spacing. 
In other words, while the larger aperture may produce a periodic lattice image that has a more impressive diffractogram, showing additional lattice reflections, the image itself is more noisy and washed out. In many cases these additional reflections are so weak that if they are filtered out, the image does not look any different. (In those rare cases where a good signal to noise ratio can be obtained, and the probe shape is known, measured and can be deconvolved, this is a legitimate method to increase the information limit (Howie, 1979; Loane et al., 1992; Shin et al., 1989; Nellist \& Pennycook, 1998; McGibbon et al., 1999).

These apertures sizes are not chosen randomly, but represent our best estimate of the experimental conditions for the ADF-STEM reported by Principe et al., (2001). The authors observed a substantial increase in the width of a gate oxide imaged by ADFSTEM compared to Cs corrected HR-TEM measurements and reconstructed electron exit waves. A close examination of the instrument used in that paper suggests that the aperture size for those experiments was probably close to $13 \mathrm{mrad}$ (semiangle). Figures 10 and 11 show that using a 13 mrad aperture leads to a larger apparent gate oxide width than with a $10 \mathrm{mrad}$ aperture. The $10 \mathrm{mrad}$ STEM results are in good agreement with the corrected CTEM measurements (Figure 11). The approximation that failed in these earlier STEM measurements was the idea that the inflexion point in the image marked the average position of the interface, independent of the shape of the probe. This is only true when the two interfaces are further apart than the probe tails. For a $2 \mathrm{~nm}$ thick oxide, this was not the case for the $13 \mathrm{mrad}$ aperture. A $10 \mathrm{mrad}$ aperture would reduce delocalization and, thereby, the measured gate width. It is worth noting that even the 10 mrad aperture would have problems accurately measuring a layer thinner than $0.5 \mathrm{~nm}$ 
- not uncommon for high-k gate dielectrics. In such cases, it would be important to either deconvolve out the point spread function, or use an aberration corrected STEM with a smaller point spread function.

How should one go about choosing the optimal aperture size? Traditionally this is calculated classically treating the diffraction blur and source size as gaussians whose widths decreases when the aperture size increased. These are balanced against a third gaussian from the blur due to the spherical aberration, which increases as the aperture size is increased (Reimer, 1997). This is not sufficiently accurate (as even a $10 \%$ error can cause problems) so numerical calculations have been performed (Colliex \& Mory, 1983; Kirkland, 1998). These calculations provide a range of aperture sizes with tolerably small delocalization to measure gate oxides thicker than $1 \mathrm{~nm}$. In reduced units the optimum aperture ADF STEM is $K=1.4$ where $K=\alpha\left(C_{s} / \lambda\right)^{1 / 4}$, with $\alpha$ being the aperture semi angle, $\underline{\lambda}$ the electron wavelength and $\mathrm{C}_{\mathrm{s}}$ the spherical aberration coefficient of the pole piece. This is typically smaller than used in bright field TEM images, $\mathrm{K}=1.5-$ 2. Increasing the STEM aperture size above optimum simply adds the extra electrons to the tails of the probe. Decreasing the aperture size below optimum does not reduce tails any further, but instead causes diffraction broadening of the central peak. This reduces the spatial resolution and beam current for no additional benefits. Tuning the aperture size can be done by adjusting the final condenser lens excitation. A quick check that the aperture is too large is if any of the circles of infinite magnification (those ring patterns used to correct probe stigmation) are visible inside the aperture. If they are visible, the aperture is too large. 


\section{Reproducible Film Thickness Determination}

Considering the issues facing TEM imaging, the prospects of arriving at a reproducible method for determining film thickness might not seem promising. In addition, preparing very thin samples is difficult and often impractical. However, ADF-STEM has an interesting advantage. It is possible to obtain high quality ADF-STEM images using relatively thick samples. Because using both HR-TEM and ADF-STEM provides more information about the interface than either method alone, we propose that the samples fall with in a range of 30 to $90 \mathrm{~nm}$. Since $90 \mathrm{~nm}$ is the upper limit of lattice imaging for phase contrast (HR-TEM), and samples thinner than $30 \mathrm{~nm}$ tend to buckle, we propose that wedge shaped samples be prepared and film thickness be determined using ADFSTEM images for sample thickness of $\sim 50 \mathrm{~nm}$. Sample thickness can be estimated using the mean free path of electrons at the probe beam energy and the ratio of the intensity of the low loss ELS signal vs. that of the probe beam. Film thickness should be reproducible under these conditions. Alternatively, sample thickness can simply be estimated from the zero beam extinction oscillation that produces intensity oscillations with sample thickness in lattice images with a periodicity of $\sim 30 \mathrm{~nm}$ at $200-300 \mathrm{keV}$ of electron energy (Pennycook \& Jesson, 1990).

A procedure for measuring pattern intensity profiles in reconstructed phase images with suitable noise suppression has been tested at the National Center for Electron Microscopy (NCEM). It allows for a direct quantitative comparison of profiles from Zand phase - contrast images as shown in Figure 11a. If the phase contrast profile \& image are aligned with the corresponding Z-contrast profile \& image at the $\mathrm{Si} / \mathrm{SiO}_{2}$ inflection point, it is seen that the phase image is in good agreement with the Z-contrast image, 
provided the optimal STEM aperture is used. When the larger than optimal aperture is used in STEM, the apparent interface width is increased by about $0.3-0.6 \mathrm{~nm}$. Thus, the observed difference relates to pattern visibility and indicates a larger sensitivity (detection limit for observation of a partial column of atoms) of the NCEM CM 300 in comparison with the non-optimal STEM, and sufficient sensitivity for both HR-TEM and optimal STEM.

Moreover, the $\mathrm{Si} / \mathrm{SiO} 2$ roughness measurement (Gaussian distributions are assumed), done at NCEM, produce 0.36 and $0.25 \mathrm{~nm} \sigma$ for the z-contrast and the phase contrast image, respectively. The different width measurements correlate with the visibility of crystal patterns. An evaluation of the poly interface is tricky in phase contrast imaging since inflection points are not necessarily recorded. However, data extrapolation to the noise limit can be used. This procedure will generate a difference in width measurements of the order of interface roughness. This leads us to make a proposal that will allow reproducible measurement of dielectric layer thickness.

We propose that microscopic measurements of dielectric layer thickness is determined from intensity traces across ADF images, following the method of Muller (2000). The data presented above from three different groups have shown that this method for thickness determination is reasonably immune to specimen thickness and interface roughness. Here we discuss the physical basis for these results, which form the scientific basis for our recommendations.

Any incoherent image is given a mathematical convolution of a specimen object function with a resolution function of the optical instrument. In the case of ADF STEM this is the intensity profile of the scanning probe (Loane et al., 1992; Pennycook \& 
Jesson, 1991; Nellist \& Pennycook, 1998; Pennycook et al., 2000). In the perfect, zone axis crystal, the specimen object function is the 1s Bloch state around each column. These columnar quantum states are the primary contribution to the contrast in a Zcontrast image. They are typically close to Gaussian in form, with a full width half maximum of $0.05 \mathrm{~nm}$ or smaller. They represent the ultimate resolution limit of the microscope (Pennycook et al., 2000). Most probes today are much broader than this. A useful concept for the present application is that of an effective probe. If the specimen is represented as an array of delta functions at each column position, the ADF image is the effective probe convoluted with the ideal delta-function object. The effective probe may be close to the incident probe in ideal circumstances, but in general will be broadened by scattering in any surface amorphous layers.

In the amorphous oxide we have no 1s Bloch states and the probe is broadened by random elastic scattering from each atom. This gives a thickness dependent probe broadening. Strictly speaking we cannot identify a unique effective probe that is independent of specimen thickness and the interface structure. The method we propose takes advantage of the fact that the effective probes are close to Gaussian in shape, both in the crystal and in the oxide provided the specimen is thin. For a Gaussian (or any radially symmetric) probe profile, a convolution with a step function (an abrupt change in intensity from $\mathrm{Si}$ to $\mathrm{SiO}_{2}$ ) will lead to a blurring of the image intensity, with the point of inflection locating the interface. Differentiating the intensity trace will regenerate the Gaussian form of the effective probe. If now the interface has structural roughness, which is usually assumed to be Gaussian in form, this will further broaden the intensity profile. However, the point of inflection will remain as the mean interface position. This 
is why the method is relatively immune to details of the specimen and microscope parameters.

Differentiating the HA-ADF intensity profile will therefore lead to a peak at the interface position (Muller, 2000). The peak width can be compared to the width of the effective probe to determine the interface roughness in thin samples. By approximating the effective probe as a Gaussian, we can now use the result that when two Gaussians are convolved, their widths add in quadrature, i.e. peak width ${ }^{2}=$ effective probe width ${ }^{2}+$ interface roughness ${ }^{2}$ ), and by inverting this relationship, estimate the interface roughness. An experimental measure of the effective probe is easily obtained by convoluting the incident probe with Gaussians of increasing width to reproduce the contrast in the Si image. The additional broadening of the derivative trace across the interface is the interfacial roughness. This procedure is illustrated in Figures 12a, b, and c. The FWHM of the derivative travel is $5.1 \AA$, and subtracting (in quadrature) the FWHM of the probe intensity profile of $1.3 \AA$ gives a roughness of $4.9 \AA$. Note, however, that the derivative trace is somewhat asymmetric. This is due to some dechanneling in the image of the Si crystal in the last few monolayers, caused by strain induced by the oxide, which is discussed further below. The distance from the peak of the derivative trace to half intensity on the oxide side of the interface is $2.0 \AA$, which if we double and subtract the probe FWHM gives an interfacial roughness of $3.7 \AA$. This illustrates the potential accuracy and errors in the method. More detailed analysis is certainly possible to better take account of the tails on the probe, the dechanneling due to strain and also the effect of specimen thickness. 
It might be asked why, if the HA-ADF image is a convolution of the object function with an effective probe, we cannot simply deconvolute the probe to retrieve the object. The reason is the limited bandwidth of the data. Deconvolution is ineffective because there is no high spatial frequency data in the image in the first place (McGibbon et al., 1999; Puetter \& Yahil, 1999). Alternative methods have been successfully applied to reconstruct objects directly from the image, including a maximum entropy method and a Pixon ${ }^{\mathrm{TM}}$ method (Ibid).

With increasing specimen thickness, the effective probe in the amorphous oxide is broadened, also, the effective probe for the crystal gains a broad background. In the case of the crystal, broadening occurs after a few tens of nm when the 1s Bloch states have been all scattered to the HA detector. This does not affect the image resolution seen with the electrons that are scattered from the first few tens of nm, but it adds a background to the image coming from electrons that propagate to greater depths before they are scattered onto the detector (Muller \& Neaton, 2001). This is the reason that the images of very thick samples still give an accurate measure of film thickness, as shown in Figure 1. Only the initial few tens of $\mathrm{nm}$ are responsible for the image contrast, and therefore allow the interface to be located to high accuracy just as in the case of a thin crystal (Muller et al., 1999; Muller \& Neaton, 2001). However, the increasing width of the effective probe with increasing thickness means that the interfacial roughness appears to increase. Therefore, measurements of interfacial roughness should be limited to samples of silicon $30 \mathrm{~nm}$ thick or less. This is shown in Figure 13. The presence of an amorphous layer on top of the sample will also broaden the beam. 
Thus, it is seen that only probe corrected ADF STEM images and reconstructed electron exit waves from instruments of comparable performance can directly be compared and may produce similar width measurement since only then direct crystal images with comparably small imaging artifacts can be produced. However, even in this case a difference will remain, since scanning noise is absent from reconstructed phase images but present in ADF STEM images and functional dependences on sample thickness, atomic numbers, and sensitivities are different. Thus, it is nothing but a matter of the materials system that dictates which method should be applied (Kisielowski et al., 2002). In the particular case of $\mathrm{Si} / \mathrm{SiO}_{2} /$ poly $\mathrm{Si}$ that is discussed here, it is the presence of the $\mathrm{SiO}_{2} /$ poly-Si interface that causes complications for HRTEM imaging. Lattice images can only be observed from grains oriented close to a zone axis of a crystal. Otherwise crystalline grains may be confused with amorphous material. Thus in HRTEM one has to make the assumption that at least one grain in the field of view is oriented such that its pattern marks the position of the $\mathrm{SiO}_{2} /$ poly $\mathrm{Si}$ interface. This depends on the poly deposition process and the resolution of the utilized instrument and could be assumed in the work of Principe et al.(2001) and Kisielowski et al.(2001; 2002). Generally however, this is not the case. In ADF STEM images, on the other hand, a contrast difference between $\mathrm{SiO}_{2}$ and poly-Si is almost always visible because of the methods larger chemical sensitivity $\left(\mathrm{Z}^{2}\right)$ and inflection points at interfaces can easily be extracted from intensity profiles even if atomic resolution cannot be obtained. Thus, even if probe correction is neglected and the resolution is limited to a typically $0.1-0.2 \mathrm{~nm}$ broad probe reproducible measurements are extractable that however may differ from instrument to instrument. 
The method we propose can be summarized as use of a 30 to $90 \mathrm{~nm}$ thick wedge polished sample and HA-ADF STEM imaging. The interface locations are determined by identification of inflection points. Another example is shown in Figure 14.

\section{Sample Preparation}

Focused Ion Beam (FIB) is a relatively new method of sample preparation that is more preferred over argon ion milling when specific sample sites must be measured. However common FIB techniques are not good for preparing TEM samples much thinner than 100 $\mathrm{nm}$ and often result in samples with gallium damaged amorphous surface layers, as thick as $20 \mathrm{~nm}$, which reduce image contrast for both HR-TEM and ADF STEM techniques. Conventional low-angle ion milling with argon can produce samples with significantly reduced surface layer damage, and recent progress has also been made by cleaning FIB samples with reduced energy gallium beams. However, the best samples we have seen tend to be those produced by wedge-polishing methods where ion milling is avoided or used only minimally and therefore we recommend such techniques until progress with surface cleaning of FIB samples is proven.

\section{Summary}

To summarize, we recommend determination of gate oxide thickness by electron microscopy from inflection points of intensity profiles in HA-ADF STEM images in samples of about 30-50 nm of thickness. These measurements are relatively insensitive to details of the specimen preparation procedure, most easily accessible and would provide well reproducible relative values. The correct selection of aperture for STEM measurement improves beam localization and results in agreement between HR-TEM and 
HA-ADF-STEM measurement of film thickness. A reconstruction of the electron exit wave also provides a direct picture of the crystal structure. However, gate thickness measurements including a $\mathrm{SiO}_{2} /$ poly $\mathrm{Si}$ interface require additional attention. Microscope parameters and recording techniques affect an absolute calibration. A determination of interfacial roughness always requires utilization of thin samples $(<20 \mathrm{~nm})$. Best results can be obtained if direct crystal images are produced by either deconvolving the probe from ADF STEM imaging or by reconstruction of aberration corrected electron exit waves. The approach we propose is the only simple way to extract quantitative measurements without extensive image simulations. The accuracy of the thickness determination has been shown to be in good agreement with thicknesses determined by bulk techniques. Differences between microscopy techniques on a scale of less than $0.3 \mathrm{~nm}$ are observed and discussed. If exploited they will reveal further details of the $\mathrm{Si} / \mathrm{SiO}_{2}$ interface.

\section{References}

Batson, P. E., Dellby N. \& Krivanek, O. L. (2002). Sub-ångstrom resolution using aberration corrected electron optics. Nature 418 617-620.

Baumann, F.H., Chang, C.-P., Grazul, J.L., Kamgar, A., Liu, C.T. \& Muller, D.A. (2000). A Closer Look at Modern Gate Oxides. Mat. Res. Soc. Symp. 611, pp. C4.1.1 C4.1.12.

Coene, W.M.J., Thust, A., Op de Beeck, M., Van Dyck, D. (1996). Maximumlikelihood method for focus-variation image reconstruction in high resolution transmission electron microscopy. Ultramicroscopy 64, 109 -135. 
Colliex, C. \& Mory, C. (1983). Quantitative aspects of scanning transmission electron microscopy, In Quantitative Electron Microscopy, Chapman, J. N. \& Craven, A. J. (Eds.) Scottish Universities Summer School in Physics pp. 149-155. Glasgow, Scotland:. IOP Pub.

Diebold, A.C., Venables, D., Chabal, Y., Muller, D., Welden, M., \& Garfunkel, E. (1999). Characterization and Production Metrology of Thin Gate Oxide and Oxynitride Films. Materials Science in Semiconductor Processing 2, 103-147.

Egerton, R.F. (1989). Electron Energy Loss Spectroscopy in the Electron Microscope. New York: Plenum.

Haider, M., Rose, H., Uhlemann, S., Schwan, E. Kabius, B. Urban, K. (1998). A spherical-aberration-corrected $200 \mathrm{kV}$ transmission electron microscope. Ultramicroscopy 75, 53-60.

Howie, A. (1979). Image contrast and localised signal selection techniques. J. Microsc. 117, 11-12.

Jia, C.L. \& Thust, A. (1999). Investigation of atomic displacements at a twin boundary in BaTiO3 by means of phase retrieval electron microscopy. Phys. Rev. Lett. 82, 5052-5055.

Kabius, B. , Haider, M., Uhlemann, S., Schwan, E., Urban, K., \& Rose, H. (2002). First application of a spherical-aberration corrected transmission electron microscope in materials science. J. Electron Microsc. 51, S51-S58.

Kirkland, E.J. (1998). Advanced Computing in Electron Microscopy. NY: Plenum. 
Kisielowski, C., Hetherington, C.J.D., Wang, Y.C., Kilaas, R., O’Keefe, M.A., \& Thust, A. (2001). Imaging columns of the light elements C, N, and O with sub-Angstrom resolution, Ultramicroscopy $\mathbf{8 9}(4), 243-263$.

Kisielowski, C., Nelson, E.C., Song, C., Kilaas, R., Thust, A. (2000). Aberration corrected lattice imaging with sub-Angstrom resolution. Microscopy and Microanalysis 6, 16-18.

Kisielowski, C., Principe, E., Freitag, B., Hubert, D. (2001). Benefits of microscopy with super resolution, Physica B 308-310, $1090-1096$.

Kisielowski, C., Jinschek, J., Mitsuishi, K., Dahmen, U. Lentzen, M. Ringnalda, J. Fliervoet, T. (2002). Exit wave reconstruction, Cs correction and Z-contrast microscopy: Comparative strengths and limitation. $15^{\text {th }}$ International Conference on Electron Microscopy, Durban, South Africa, p. 165-167.

Krivanek, O.L., Dellby, N. \& Lupini, A.R. (1999). Towards sub-angstrom electron beams. Ultramicroscopy $\mathbf{7 8}, 1-11$.

Loane, R.F., Xu,P. \& Silcox, J. (1992). Incoherent imaging of zone axis crystals with ADF STEM. Ultramicroscopy 40, 121-138.

McGibbon, A.J., Pennycook, S.J., \& Jesson, D.E. (1999). Crystal structure retrieval by maximum entropy analysis of atomic resolution incoherent images. J. Microsc. 195, $44-57$.

Muller, D. A. (1998). Alternatives to Core-Loss Compositional Imaging,. In Proc. of 14th Int'l. Congress on Electron Microscopy, pg 219-220. Bristol, UK: Institute of Physics Publishing. 
Muller, D.A., Sorsch, T., Moccio, S., Baumann, F.H., Evans-Lutterodt, K. \& Timp, G. (1999). The electronic structure at the atomic scale of ultrathin gate oxides. Nature 399, $758-761$.

Muller, D.A. (2000). Gate Dielectric Metrology Using Advanced TEM Measurements. In Characterization and Metrology for ULSI Technology 2000, Seiler, D.G., Diebold, A.C., Shaffner, T.J., McDonald, R., Seiler, D.G., Diebold, A.C., Shaffner, T.J., McDonald, R., Bullis, W.M., Smith, P.J., and Secula, E.M., (Eds.) pp 500-505. Melville, NY: AIP Press.

Muller, D.A. \& Neaton, J.D. (2001). Evolution of the Interfacial Electronic Structure During Thermal Oxidation, In Fundamental Aspect of Silicon Oxidation, Y. Chabal, (Ed.), pp. 219-246. New York: Springer.

Nellist, P.D. \& Pennycook, S.J. (1998). Sub-angstrom resolution by underfocused incoherent transmission electron microscopy. Phys. Rev. Lett. 81, 4156-4159.

Nellist P.D. \& Pennycook S.J. (1998). Accurate structure determination from image reconstruction in ADF STEM. J. Microsc. $190,159-170$.

O’Keefe, M.A., Herington, C.J.D., Wang, Y.C., Nelson, E.C., Turner, J.H., Kisielowski, C., Malm, J.-O., Mueller, R., Ringnalda, J., Pan, M., Thust, A. (2001). SubAngstrom high resolution transmission electron microscopy at $300 \mathrm{keV}$, Ultramicroscopy, 89(4), 215-241.

Pantel, R., Sondergard, E., Delille, D. \& Kwakman, L.F.Tz. (2001). Quantitative Thickness Measurements of Thin Oxides Using Low Energy Loss Filtered TEM Imaging. Microsc. \& Microanal. Vol 7 (Suppl 2) pp 560-561. 
Pantelides, S.T., Ramamoorthy, M., Rashkeev, S., Buczko, R., Duscher, G. \& Pennycook, S.J. (2001). Local and Global Bonding at the Si-SiO2 Interface. In Fundamental Aspects of Silicon Oxidation. Chabal, Y. J. (Ed.), 193-218, Springer Series in Materials Science. Berlin: Springer-Verlag.

Pennycook, S. J. \& Boatner, L. A. (1988). Chemically Sensitive Structure Imaging with a Scanning Transmission Electron Microscope Nature 336, 565-567.

Pennycook, S. J. \& Jesson, D. E. (1990). High-resolution incoherent imaging of crystals Phys. Rev. Lett. 64, 938-941.

Pennycook, S.J. \& Jesson, D.E. (1990). High-resolution incoherent imaging of crystals. Phys Rev Lett, 64, 938-941.

Pennycook, S. J. \& Jesson, D. E. (1991). High-resolution Z-contrast imaging of crystals. Ultramicroscopy, 37, 14-38.

Pennycook, S.J. (1997). Scanning Transmission Electron Microscopy : Z-Contrast. In Handbook of Microscopy, Amelinckx, S., Van Tendeloo, G., Van Dyck, D. \& Van Landuyt, J. (Eds.), pp. 595-620. Weinheim, Germany: VCH Publishers.

Pennycook, S. J., Rafferty, B. \& Nellist, P. D. (2000). Towards Z-Contrast Imaging in an Aberration-Corrected STEM. Microsc. Microanal. 6 (Suppl 2), 106-107.

Pennycook, S.J., Lupini, A.R. \& Nellist, P.D. (2002). The Ultimate Resoltuion in Abberation-Corrected STEM. Microsc. Microanal. 8 (Suppl 2), 16-17.

Perovic, D.D., Rossow, C. J. \& Howie, A. (1993). Imaging elastic strains in high-angle annular dark field scanning transmission electron microscopy. Ultramicroscopy $\mathbf{5 2}$, $353-359$. 
Puetter, R.C. Yahil, A. (1999). In ASP Conf. Ser., Vol 172, Astronomical Data Analysis Software and Systems VIII, Mehringer, D.M., Plante, R.L. \& Roberts, D.A. (Eds.), pp 307-316. San Francisco: ASP.

Principe, E., Hegedus, A., Chua, T.C. \& Olson, C. (2001). Hyper Thin Nitrided Gate Oxide Characterization Methodology, presented at the Quantitative Surface Analysis Conference, San Jose: AVS.

Principe, E., Watson D.G., Kisielowski, C. (2002). Advancements in the Characterization of "Hyper-Thin" Oxynitride Gate Dielectrics Through Exit Wave Reconstruction HRTEM and XPS, in Microelectronic Failure Analysis Desk Reference 2002 Supplement, (Eds.) pp59-68, Materials Park, Ohio: ASM International.

Rau, W.D. \& Lichte, H., (1999). High resolution off-axis electron holography, in Introduction to Electron Holography, Volkl, E., Allard, L.F. \& Joy, D.C. (Eds.) pp 201 -229. New York: Kluwer Academic.

Reimer, L. (1997). Transmission Electron Microscopy: Physics of Image Formation and Microanalysis, Springer Series in Optical Science Volume 36. New York: Springer.

Ross, F.M. \& Stobbs, W.M. (1991). A study of the initial stages of the oxidation of silicon using the Fresnel Method, Philosophical Mag. A 63, 1-36.

Shin, D.H., Kirkland, E.J. \& Silcox, J. (1989). Annular dark field electron microscope images with better than $2 \AA$ resolution at $100 \mathrm{kV} \mathrm{Appl.} \mathrm{Phys.} \mathrm{Lett.} \mathrm{55,} \mathrm{2456-2458.}$

Silcox, J., Xu, P. \& Loane, R. L. (1992). Resolution limits in annular dark field STEM . Ultramicroscopy 47, 173-186.

Taylor, S. Mardinly, J. O'Keefe, M.A. \& Gronsky, R. (2000). HRTEM Image Simulations for Gate Oxide Metrology, In Characterization and Metrology for 
ULSI Technology 2000. , Seiler, D.G., Diebold, A.C., Shaffner, T.J., McDonald, R., Bullis, W.M., Smith, P.J., \& Secula, E.M. (Eds.) pp 130-133. AIP conference Proceedings 550. Melville, NY: AIP Press.

Thust, A., Coene, W.M.J., Op de Beeck, M., Van Dyck, D. (1996). Focal-series reconstruction in HRTEM: simulation studies on non-periodic objects . Ultramicroscopy 64, 211-230.

van Dyck, D., \& Chen, J.H. (1999). A simple theory for dynamical electron diffraction in crystals. Solid State Communications 109, 501-505.

Wang, Y.C., Fitzgerald, A., Nelson, E.C., Song, C., O’Keefe, M.A. \& Kisielowski, C. (1999). Correction of the 3 -fold astigmatism and lattice imaging with information below 100 pm. Microscopy and Microanalysis 5, 822-824.

Wang, Z. L. (1997). Lattice imaging using plasmon energy-loss electrons in an energyfiltered transmission electron microscope. Ultramicroscopy 67, 105-111. 


\section{Figure Captions}

Fig. 1. A constant layer thickness to within $0.1 \mathrm{~nm}$ is observed for a $2 \mathrm{~nm}$ silicon dioxide layer when 600, 550, and $300 \mathrm{~nm}$ thick samples are imaged by HA-ADF-STEM.

Figure published previously by Muller (2000).

Fig. 2. Lattice fringes in HR-TEM appear at interfaces that can make rough interfaces appear smooth. Defocus and sample thickness dictate the apparent abruptness.

Fig. 3. The effect of interfacial microroughness on a single lattice image is illustrated by the simulation of Baumann, et al., (2000). The periodicity of the simulated roughness is shown in the upper left. The blue areas are indentations in the interfacial plane, and the red areas are elevations. The right column of images are simulated results for conventional HR-TEM or bright field STEM.

Fig. 4. (a) Exit wave multislice simulation of the interface model from figure 3 for a 200 $\mathrm{kV}$ TEM with $\mathrm{Cs}=0 \mathrm{~mm}$ and no noise. (b) ADF image for a $200 \mathrm{kV}$ STEM with $\mathrm{Cs}=$ $0.2 \mathrm{~mm}$. In both cases the sample is $7.6 \mathrm{~nm}$ thick.

Fig. 5. Line profiles through the calculated exit wave (dashed line) and ADF-STEM (solid line) images from figure 4 for a $7.6 \mathrm{~nm}$ thick sample, both the ADF and exit wave images reproduce the projected potential for thin samples in the absence of noise. Image intensities are smaller in ADF-STEM and scaled to phase contrast values.

Fig. 6. Comparison of HR-TEM (CM300 FEG OAM, Cs $=0.6 \mathrm{~mm} 300 \mathrm{kV})$ and HAADF STEM (JEOL 2010F, Cs $=1 \mathrm{~mm} 200 \mathrm{kV}$ ) images of the same silicon oxynitride sample. The silicon substrate is on the right, and the polysilicon capping layer(deposited after XPS analysis) is on the left. (a) The HA-ADF STEM is sensitive to density differences in amorphous materials. The oxynitride layer (in the middle of 
the image) has decomposed into three bands, with two lower density regions at the oxide/silcon interface. It is possible that during the polysilicon depostion, the interfaces have become oxidized. (b) an exit wave reconstruction of a HR-TEM image. The interface between the oxide and the polysilicon is not always apparent and contrast between different amorphous materials and non-oriented crystallites (eg. In the polysilicon) is poor. Inset is an XPS depth profile on the oxynitride film prior to polysilicon deposition.

Fig. 7. Image blur and delocalization for ADF-STEM and HR-TEM (lattice images) in a $200 \mathrm{keV}$ instrument with $\mathrm{Cs}=1 \mathrm{~mm}$, (a) The point spread functions (PSF) for ADFSTEM and phase contrast lattice images (inverted for comparison) showing a narrower central peak, smaller side lobes and no contrast reversals for STEM (10 mrad probeforming aperture in STEM, 0.1 mrad beam divergence in HR-TEM, $45 \mathrm{~nm}$ defocus for both). (b) The data of (a) plotted on a log scale showing the one to two order of magnitude reduction in probe tails at large distances for ADF-STEM. (c) The effect of increasing the probe forming aperture in STEM (13 vs. $10 \mathrm{mrad})$ is to increase the strength of probe tails i.e. the delocalization.

Fig. 8. The contrast transfer function for ADF-STEM at $200 \mathrm{kV}$ and $1 \mathrm{~mm}$ Cs for two different sizes of the probe-forming aperture. The $10 \mathrm{mrad}$ aperture is close to optimal (as described in the text). While the $13 \mathrm{mrad}$ aperture does provide an increased information limit, it also reduces the contrast at the lower spatial frequencies, sometimes by as much as a factor of two. This will not only lead to a noisier image of the $\{111\}$ silicon lattice, but also will increase image delocalization. 
Fig. 9. Probe tails for 2 different sizes of the probe forming aperture at $200 \mathrm{kV}$ and $1 \mathrm{~mm}$ Cs. Increasing the aperture by $30 \%$ increases the radius containg $80 \%$ of the signal from $0.25 \mathrm{~nm}$ to $0.8 \mathrm{~nm}$. All the extra beam current added by the large aperture is in the probe tails. This increases the background, but not the localized signal. This is a problem not only for ADF, but also for EELS and EDX (which again depend on the probe shape).

Fig.10. The same nitrided gate oxide imaged by different methods. (a) EWR (b) ADFproper size aperture of $10 \mathrm{mrad}(\mathrm{JEOL})$ c) ADF-13 mrad aperture too big (JEOL) d) ADF aperture too big (FEI).

Fig. 11. (a) Direct comparison of HR-TEM and ADF-STEM measurement of dielectric layer thickness. For details see text. (b) Line profiles of ADF-STEM images of the same gate oxide, but with two different probe-defining aperture sizes. The $13 \mathrm{mrad}$ aperture causes large image delocalization and oxide width measured from the inflexion points is $2.49 \mathrm{~nm}$. The $10 \mathrm{mrad}$ aperture has minimal delocalization and the measured width is $2.13 \pm 0.08 \mathrm{~nm}$, which is within experimental error of the HR-TEM measured thickness after exit wave reconstruction.

Fig. 12. HA-ADF image of a $\mathrm{Si}-\mathrm{SiO} 2$ interface showing extraction of interface roughness. The image is obtained with a VG Microscopes HB603U dedicated STEM with a probe size of about $0.13 \mathrm{~nm}$, sufficiently small to resolve the dumbbells. A vertically averaged line trace (grey) is fitted with a smooth curve (dashed line), differentiated (dotted line) and the width compared to the probe profile.

Fig. 13 Schematic showing how columns in a zone axis crystal act to channel the beam and delay the beam broadening that occurs in an amorphous or randomly oriented 
crystal. a) Thin samples. b) With increasing thickness the crystal image also acquires a background due to broadening, contrast reduces but the resolution remains.

Fig. 14 The thickness of a thin silicon dioxide layer is determined using HA-ADFSTEM. 